\title{
When the going gets tough
}

In 1995, a meeting at the Los Alamos National Laboratory in New Mexico brought together a hundred or so scientists to explore a metaphor that could well have been inspired by the chaotic desert landscape of the area. The meeting, entitled Landscape Paradigms in Physics and Biology, focused on the rugged structural complexity of conceptual landscapes - of energy, biological fitness and so on - in systems where conflicting constraints or interactions prevent the system from finding its way easily to some global minimum or maximum (depending on the situation), and make a host of local minima or maxima much more relevant to the observed behaviour.

The promising idea, then, was that a good deal might be understood about systems in biology, physics, perhaps even economics, by teasing out the generic implications of such landscape roughness and complexity. This had already proved to be true for physical systems, especially spin glasses or glassy materials, and for the dynamics of neural networks, as well as in evolutionary biology, where individual fitness may be a key principle but every species exhibits once-adaptive but now suboptimal features locked in place by evolution.

More controversial was the idea that thinking about rugged landscapes might help explain the puzzling physics and chemistry of protein folding. If the physicists present were excited about the possibility, the biologists were rather less so. The dynamics of protein folding should follow from the landscape of the free energy of the amino-acid chain as a function of its configuration. Fifteen years later, it's still not clear how much the key aspects of the problem - folding speed, for example, and the character of the folded, native structures - depend on the complexity of such landscapes, but recent experiments suggest it may be very important indeed.

One of the new ideas back in 1995 was that the free-energy landscape of a protein must have a funnel-like shape. If a protein explored its configuration space at random, it would never fold on realistic timescales given the astronomical number of conformations possible, even for a small protein. One way out of this paradox, as suggested originally by Peter Wolynes et al., is that the interactions among the amino acids in proteins must have a delicate organization that guarantees an overall funnel-like shape to the free-energy landscape, which effectively guides the protein more or less smoothly towards the correct folded state.

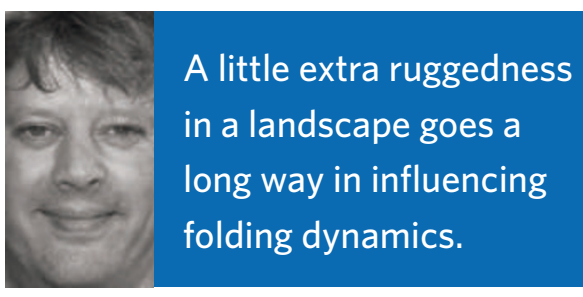

This idea is now well established, particularly on the basis of extensive simulations mapping out the free-energy landscapes for large polymer chains. Most polymers with random sequences don't fold well; the sequences of the small fraction that do fold well (quickly and to a stable state) lead to funnel-like free-energy surfaces. But other questions remain unanswered, in particular whether the ruggedness of protein energy landscapes - undulations on small scales plays a part in slowing a protein's folding.

Beth Wensley and colleagues now have evidence that roughness does slow down the folding, from the folding kinetics of the R15, R16 and R17 domains of the cytoskeletal protein spectrin (Nature 463, 685-688; 2010). The similar structures and stability of these three domains would suggest that they should fold very similarly as well. Yet R15 folds 3,000 times faster than the other two. Why?

The reason, Wensley et al. suggest, lies in the pronounced ruggedness of the landscape on a fine scale for R16 and R17, which creates a kind of 'internal friction' in their folding dynamics. The folding rate of a protein can be analysed as a diffusive process on a landscape, and a standard calculation shows the overall rate of folding to be inversely proportional to the sum of two dissipative forces slowing it down: the viscosity of the surrounding solvent and the effective internal friction of the protein. If the internal friction were small compared with that of the solvent, then a plot of rate versus solvent viscosity should simply be linear, which is precisely what these authors found in the case of R15, the fast folder.

But similar plots show very different results for R16 and R17, and suggest the influence of internal friction that is about four or twelve times higher, respectively. From this data the authors were also able to estimate the rough size of the small-scale ruggedness in the free-energy landscape that would lead to such friction, finding that an increase in the typical energy barrier size by a factor of about two is enough to account for it. Hence, it seems that a little extra ruggedness in a landscape goes a long way in influencing folding dynamics. In more specific terms, Wensley and colleagues conjecture that the cause of this enhanced ruggedness is a tendency, in R16 and R17, for long helices to mis-dock in various positions during the fold, something that doesn't happen for R15.

Perhaps an even more striking result showing the importance of landscape complexity comes from experiments on RNA polymers by Sergey Solomatin et al. (Nature 463, 681-684; 2010). Biologists generally assume that the sequence of a biological macromolecule specifies its folded, native structure in a unique way as a single, global energy minimum. But the enormous complexity of such landscapes especially for large macromolecules - makes it at least theoretically possible that some polymers might not have a single global minimum, but rather a number of deep local minima corresponding to different native conformations. Some experiments have shown tentative evidence for such states in proteins.

For RNA, Solomatin et al. have shown very clearly that this is the case in the context of a ribozyme of the protozoa Tetrahymena an RNA enzyme of some 400 nucleotides that folds under the right conditions into an active structure able to catalyse the cleavage of certain oligonucleotides. In careful experiments using single molecules, they found that these proteins don't always fold into the same native state; rather, the final state of the protein seems to be one of about seven different possible structures showing a variation in their activity as enzymes. More importantly, they also found that these structures could interconvert on a timescale of the order of seconds, changing from one form to another.

This is among the clearest evidence yet for the potentially enormous importance of landscape complexity in molecular biology, where it may in many cases even undermine the existence of a unique folded state. No doubt if this turns out to be widespread, it will also have been exploited by biology for some purpose - perhaps in allowing a greater repertoire of functions to be carried out by a single biopolymer.

Even after 15 years, it seems that the full value of this metaphor is still not clear. Metaphor has a unique power in science, as an engine of creative ideas, but it runs well ahead of the experiments that are able to test them. $\square$

MARK BUCHANAN 